\title{
Editorial: Advance of simulations and techniques for communication networks and information systems
}

\author{
Dingde Jiang ${ }^{1} \cdot$ Houbing Song ${ }^{2} \cdot$ Liuwei Huo $^{1}$
}

Accepted: 10 March 2021 / Published online: 16 April 2021

(C) The Author(s), under exclusive licence to Springer Science+Business Media, LLC, part of Springer Nature 2021

Nowadays, following the successful application of wireless communication and the fast development of the fifth generation communication $(5 \mathrm{G})$, a large number of new emerging applications and services, such as smart city, smart transportation, smart grid, smart home, smart medical, smart agriculture and unmanned driving, have brought great challenges to the current network. They put forward higher requirement on the network, such as throughput, access volume, latency and energy efficiency. In order to satisfy the requirement of new emerging applications and services, a large number of new technologies, such as device-to-device (D2D), wireless relay, software defined network (SDN), network function virtual(NFV), network slicing, edge computing, cloud computing, machine learning and swarm intelligent algorithm, are deployed into the network. How to effectively apply and benefit from the above technologies and make them intelligently interoperate together? How to simulate the behavior of services and verify the functions of applications in the network? Obviously, integrating all above existing advanced technologies, modeling and simulating the behavior of services and verifying the functions of applications is a huge challenge in both research and development and gains great attention from academics and industries.

This special issue focuses on a broad range of research in the field of simulation, modeling and analysis, and aims to address current and future trends in simulation, models, practices and software. This special issue features twenty selected papers with high quality.

Dingde Jiang

jiangdd@uestc.edu.cn

1 School of Information and Communication Engineering, University of Electronic Science and Technology of China, Chengdu 611731, China

2 Department of Electrical, Computer, Software, and Systems Engineering, Embry-Riddle Aeronautical University, Daytona Beach, FL 32114, USA
The first article "Network Recovery for Large-Scale Failures in Smart Grid by Simulation" by Huibin Jia, et al. proposed an importance-based recovery method for largescale failure in the smart grid to overcome the large-scale natural disaster or malicious attacks which could cause serious damage to the power communication network in smart grid.

Uyghur is a language widely used in Xinjiang, and the Uyghur speech recognition and text recognition are of great significance to the education and inheritance of Uyghur. The second article titled "Analysis of Phonemes and Tones Confusion Rules Obtained by ASR" by Gulnur Arkin, et al. explored the effective method of erroneous phoneme pronunciation of Chinese mandarin learners whose mother tongue is Uyghur and the solution of major problems of language education, concerning the learner's pronunciation. Then, authors proposed the Automatic Speech Recognition (ASR) to recognize phonemes of the pronunciation of Chinese mandarin learners.

In the third article, "An Adaptive Threshold Algorithm for Offline Uyghur Handwritten Text Line Segmentation" presented an effective text-line segmentation algorithm and evaluated its performance on Uyghur handwritten text document images. In this algorithm, the projection based adaptive threshold selection mechanism is implemented to detect and segment the text lines with different valued thresholds.

Indoor Location technology is usually used as an auxiliary positioning for satellite positioning, to solve the problem that the satellite signal is weak when it reaches the ground and cannot penetrate the building. In the fourth article, "Bisecting K-Means Based Fingerprint Indoor Localization" proposed an indoor localization system based on Bisecting k-means (BKM). In this method, the BKM-based indoor localization method is divided into two stages: offline stage and online positioning stage. In the offline stage, the BKM is used to divide all the reference points into $\mathrm{k}$ clusters. 
Image encryption is a technology that uses the characteristics of digital images to design encryption algorithms to improve encryption security and operational efficiency. In the fifth article with the title "Computer Generated Hologram-Based Image Cryptosystem with Multiple Chaotic Systems", the authors present a novel image encryption scheme based on computer generated hologram (CGH) and multiple chaotic systems. In this method, authors combined the positions and changing the values of image pixels to confuse the relationship between the cipher text and the original image.

In the sixth article with the title "HSS-Iteration-Based Iterative Interpolation of Curves and Surfaces with NTP Bases", the authors proposed a new PLA approach based on the HSS iterative method for solving linear equations to solve the problems of curves and surfaces interpolation with normalized totally positive (NTP) bases. Then, authors considered the weights to speed up the convergence rate of the iterative process, namely WHPIA, and gave the approximate value of the fastest convergence weight.

Slow convergence and low efficiency are the problems faced by existing adaptive PID controllers. The seventh article, "The Adaptive PID Controlling Algorithm Using Asynchronous Advantage Actor-Critic Learning Method" proposed a new adaptive PID controller using the Asynchronous Advantage Actor-Critic (A3C) algorithm to address the problems of the slow convergence and inefficiency in the existing adaptive PID controllers. In the method, the controller can train the multiple agents of the actor-critic structures in parallel exploiting the multi-thread asynchronous learning characteristics of the A3C structure, and each agent uses a multilayer neural network to approach the strategy function and value function to search the best parameter-tuning strategy in continuous action space.

In the next article with the title "Exploring MDE Techniques for Engineering Simulation Models", the authors studied the model-driven engineering (MDE) to tackle the ever-increasing complexity of simulation system, and presented the contributions in two main aspects: customizing domain specific language by metamodeling and enhancing model continuity by formalizing model transformations.

Swarm intelligence algorithms are algorithms inspired by the behavior of animals in the nature. They are widely used to solve the optimization problems. The Artificial bee colony $(\mathrm{ABC}$ ) algorithm is a swarm intelligence algorithm that imitated bees foraging behavior. The ninth article, "An Improved Exhausted-Food-Sources-Identification Mechanism for the Artificial Bee Colony Algorithm" designed an improved exhausted-food-source-identification mechanism based on space partitioning which considered the food source states both in the objective space and in searching space simultaneously.

In the tenth article with the title "A Knee Point-Driven Multi-objective Artificial Flora Optimization Algorithm", the authors proposed a knee point-driven MOAF (kpMOAF) optimization algorithm to address the vulnerability of MOAF optimization algorithm. This method take the knee points of the non-dominant solutions as criterion to guide the population evolution.

Network selection is a very critical step when users access the network. In the eleventh article "A QoE-Based Dynamic Energy-efficient Network Selection Algorithm", the authors studied a QoE based dynamic network selection algorithm. In this method, the authors proposed to use the online dynamic learning property of Q-learning method considering users' QoE, networks' energy consumption, and switch times together.

Medical image processing is one of the most critical technologies in telemedicine. In the twelfth article titled "An Improved Image Registration and Fusion Algorithm", the authors proposed a new registration and mosaic algorithm for medical images to solve the problems of electronic noise, uneven illumination and ray scattering in the real-time medical process.

The user behavior characteristics of mobile social network services are of guiding significance to the evaluation of user experience. In the thirteenth article with the title "User Behavior and User Experience Analysis for Social Network Services", the authors studied the amount of user action triggered by the communication angle and the fluctuation of the user's action communication performance under different scenarios. In this article, the authors analyzed the distribution of data concerning different user actions and tested the waiting time and success rate of different user actions in different scenes.

In the fourteenth article, "Discriminative Matrix-variate Restricted Boltzmann Machine Classification Model" proposed the improved MVRBMs for classification and used it to classify 2D data directly and accurately. In this method, authors added the classification constraint to MVRBM to get Matrix-variate Restricted Boltzmann Machine Classification Model (ClassMVRBM), and proposed fisher discriminant analysis criterion for matrix-style variable and applied to the hidden variable.

Optical network simulation is very important for optical network optimization. In the fifteenth article with the title "ITU TWDM-PON Module for NS-3", the authors proposed an ITU TWDM-PON module for the NS-3 network simulator based on the XG-PON module, and described its concept and design specifications. The proposed module can simulate G.989 standard-compliant data packet transmission in the upstream and down- stream directions using multiple wavelength channels. 
The sixteenth article, "Development of An Effective Method of Tariff Formation for Rural Areas: The Case of Russian Federation" studied the tariff formation for rural areas. The authors found that none of the current pricing and tariffs' setting methods fulfills the requirements for an effective and economically founded tariff policy in the housing and communal services sector, and developed a new methodology that will ensure the receipt of compromise tariffs for housing and communal services.

The development of Unmanned Aerial Vehicle (UAV) technology is very rapid and has been highly praised by the industry and academia. The flight Mach controller as a necessity of an aircraft has become one of the research hotspots. In the seventeenth article "Study and Hardwarein-the-loop Simulation of Flight Mach Control System Based on Fuzzy Control Theory", the authors designed a Flight Mach Fuzzy Controller (FMFC) based on modern Fuzzy control theory for an UAV using a turbojet engine, and optimized the control performance by changing the scaling factor. Finally, authors carried out the mathematical simulation and hardware-in-the-loop simulation to evaluate the control performance.

Software-defined networking (SDN) separates the control plane from the data plane of network devices and realizes programmability of network behavior. It is recognized as one of the most promising technologies for future core network. In power communication networks based on SDN, the measurement and prediction of network traffic is the key to network management. In the eighteenth article "A SDN-Based Intelligent Prediction Approach to Power Traffic Identification and Monitoring for Smart Network Access", the authors combined the Software Defined Networking (SDN) architecture and Radial Basis Function neural network (RBFNN) for traffic intelligent prediction in the power information communication network (PICN). In this method, the complex nonlinear relationships of large-scale network traffics are analyzed by RBFNN model to realize high-precision traffic identification.

The next article with the title "A SDN-Based Active Measurement Method to Traffic QoS Sensing for Smart Network Access" proposed a SDN-based active measurement method to measure the evaluation parameters of the smart network access in the power communication network. In this method, authors collect statistics from OpenFlow switches and defined the parameters that should be measured; Finally, the authors proposed an algorithm to updated the measurement interval based on the measurement throughput changes.

Energy is the most valuable resource in D2D relay networks. High energy efficiency can effectively extend the survival time of D2D relay networks. The last article titled "Energy Efficiency Resource Allocation for D2D
Communication Network Based on Relay Selection" studied a new model that combines with D2D communication and energy harvesting technology. In this model, authors investigated the maximization energy efficiency (EE) of the network by joint time allocation and relay selection while taking into the constraints of the signal-tonoise ratio of D2D and the rates of the Cellular users, and the maximization problem of EE was divided into two subproblems: relay selection problem and time optimization problem.

Acknowledgements The guest editors are thankful to our reviewers for their effort in reviewing the manuscripts. We also thank the Editin-Chief, Dr. Imrich Chlamtac for his supportive guidance during the entire process. The special issue is sponsored by the National Natural Science Foundation of China (No. 61571104), the Sichuan Science and Technology Program (No. 2018JY0539), the Key projects of the Sichuan Provincial Education Department (No. 18ZA0219), the Fundamental Research Funds for the Central Universities (No. ZYGX2017KYQD170), the CERNET Innovation Project (No. NGII20190111), the Fund Project (Nos. 61403110405, 315075802, JZX6Y202001010161), and the Innovation Funding (No. 2018510007000134). Dr. Dingde Jiang is corresponding author of this special issue (email: jiangdd@uestc.edu.cn).

Publisher's Note Springer Nature remains neutral with regard to jurisdictional claims in published maps and institutional affiliations.

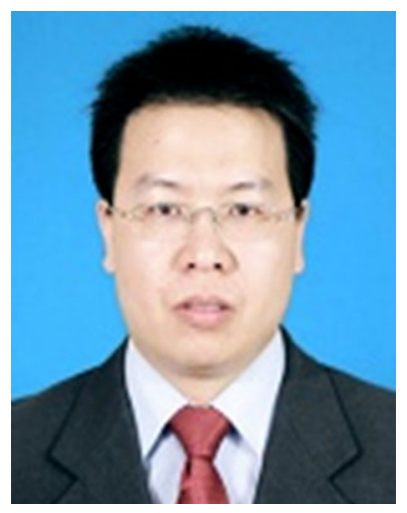

Dingde Jiang (M'08) is currently a Professor with University of Electronic Science and Technology of China. His research focuses on network measurement, modeling and optimization, performance analysis, network management, network security in communication networks, particularly in software-defined networks, information-centric networking, energy-efficient networks, and cognitive networks. His research is supported by the National Science Foundation of China, the Program for New Century Excellent Talents with the University of Ministry of Education of China, and so on.Dr. Jiang served as a technical program committee member for several international conferences. He has received the best paper awards at several international conferences. He has been serving as an editor for one international journal.. 


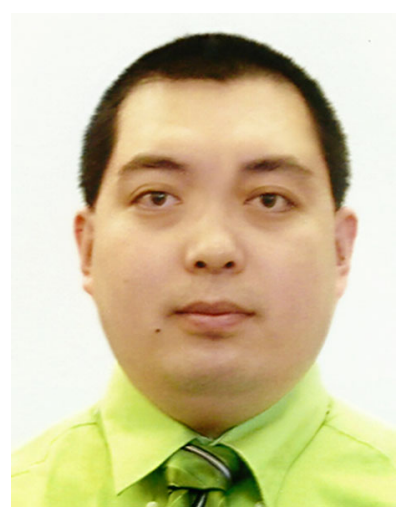

Houbing Song (M'06-SM'15) is received the Ph.D. degree in electrical engineering from the University of Virginia, Charlottesville, VA, in August 2012.In August 2017, he joined the Department of Electrical, Computer, Software, and Systems Engineering, Embry-Riddle Aeronautical University, Daytona Beach, FL, where he is currently an Assistant Professor and the Director of the Security and Optimization for Networked Globe Laboratory (SONG Lab, www.SONGLab.us). He served on the faculty of West Virginia University from August 2012 to August 2017. In 2007 he was an Engineering Research Associate with the Texas A\&M Transportation Institute. He serves as an Associate Technical Editor for IEEE Communications Magazine. He is the editor of four books, including Smart Cities: Foundations, Principles and Applications, Hoboken, NJ: Wiley, 2017, Security and Privacy in Cyber-Physical Systems: Foundations, Principles and Applications, Chichester, UK: Wiley-IEEE Press, 2017, Cyber-Physical Systems: Foundations, Principles and Applications, Boston, MA: Academic Press, 2016, and Industrial Internet of Things: Cybermanufacturing Systems, Cham, Switzerland: Springer, 2016. He is the author of more than 100 articles. His research interests include cyber-physical systems, cybersecurity and privacy, internet of things, edge computing, big data analytics, unmanned aircraft systems, connected vehicle, smart and connected health, and wireless communications and networking.Dr. Song is a senior member of ACM. Dr. Song was the very first recipient of the Golden Bear Scholar Award, the highest campus-wide recognition for research excellence at West Virginia University Institute of Technology (WVU Tech), in 2016.

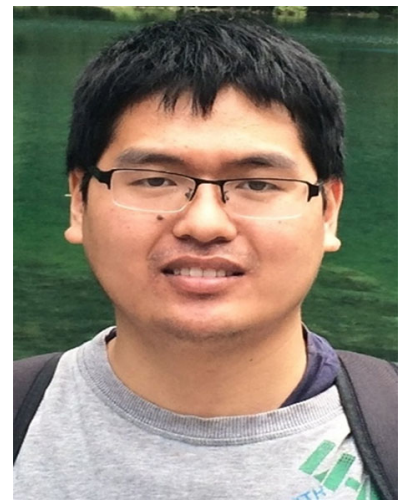

Liuwei Huo $\left(M^{\prime} 08\right)$ received the M.S. degree in communication and information systems from the Chongqing University of Posts and Telecommunications, Chongqing, China, in 2016. He is currently working for his $\mathrm{Ph} . \mathrm{D}$ degree in Communication and Information System, Northeastern University, China. Currently, he is also a visting student in University of Electronic Science and Technology of China, Chengdu, China. His research interests include cooperation communication, software defined networks and network measurement. 